\title{
Magnetic Moments' Arrangement in Fe Layers Deposited from Acetone Based Electrolyte
}

\author{
W. Olszewski ${ }^{a, *}$, K. Szymański ${ }^{a}$, D. SAtula ${ }^{a}$, \\ M. BIERNACKA ${ }^{a}$ AND E.K. TALIK ${ }^{b}$ \\ ${ }^{a}$ Faculty of Physics, University of Białystok \\ Lipowa 41, 15-424 Białystok, Poland \\ ${ }^{b}$ August Chełkowski Institute of Physics, University of Silesia \\ Uniwersytecka 4, 40-007 Katowice, Poland
}

The acetone based electrolyte was used for electrodeposition of iron layers on the copper substrate. Two types of surfaces of the deposited layer can be obtained: shiny or black. Magnetic properties of the near-surface regions were studied by the conversion electron Mössbauer spectroscopy. The conversion electron Mössbauer spectra revealed apparent dependence of magnetic moments' arrangement on the deposition time. Those results were compared with the magnetization measurements. Composition of black coating was examined by the X-ray photoelectron spectroscopy measurements.

PACS numbers: 81.15.Pq, 33.45.+x, 76.80.+y, 75.30.Gw, 75.30.Cr

\section{Introduction}

Layers of iron and its alloys have wide range of application, especially in the electronic industry $[1,2]$ because of their desirable magnetic properties such as high values of saturation magnetization and coercive force [3] as well as the possibility of preparation samples with different types of anisotropy [4]. Electrodeposition is one of the cheapest and convenient method of synthesis of metallic layers. In most cases the electroplated iron layer is achieved from acidic electrolytes such as sulphate [5], chloride [6] and sulfamate [7]. There were also some attempts to electroplate iron layers from gluconate baths [8]. However, in this case, the surface morphology was not of the best quality.

Practically, all commercial baths contain many components in order to improve coating characteristics, such as microstructure or surface morphology. Thus,

${ }^{*}$ corresponding author; e-mail: wojtek@alpha.uwb.edu.pl 
a multicomponent electrolyte is designed for rather limited compositions of the deposited layers.

Complex composition of electroplating baths motivated us to search for simple methods of deposition. We developed a method of electroplating shiny layers (Fe, $\mathrm{Co}, \mathrm{Ni}, \mathrm{Cu}$ and $\mathrm{Zn}$ ) from a new type of electrolyte. At the beginning of the process, the bath contains only acetone, a small amount of water and hydrochloric acid. Under dc current mode the anode dissolves and finally the metallic layer is deposited. Layers are well connected to the cathode substrate.

The aim of the presented contribution was to obtain iron layers by electrodeposition from acetone based bath and to determine some of their magnetic properties.

\section{Details of layer deposition}

The electrolyte contains: $94.8 \%$ of acetone, $0.8 \%$ of concentrated hydrochloric acid ( $36 \%$ solution of $\mathrm{HCl}$ in water) and $4.4 \%$ of water. The solution temperature was maintained at $295 \mathrm{~K}$.

The anode was made of iron (purity 99.9\%). The cathode was a disk of copper (purity 99.9\%) with exposed surface area $4 \mathrm{~cm}^{2}$ placed on the bottom of the electrochemical cell. Before electrodeposition, in order to have controlled conditions, the cathode was mechanically polished. Then it was electropolished in acid solution for $5 \mathrm{~min}$ and rinsed in acetone. These treatments were performed just before the substrate immersion, in order to avoid exposure to atmospheric oxygen.

Electrodeposition experiments were carried out using dc current mode. The density of cathodic current was stabilized to $5 \mathrm{~mA} / \mathrm{cm}^{2}$. The time of the deposition $\left(t_{\mathrm{D}}\right)$ was varied from 5 to $20 \mathrm{~min}$. Thickness of layers was determined by scanning electron microscopy (SEM) measurements [9]. SEM results indicate that layers, with $t_{\mathrm{D}}=15 \mathrm{~min}$, are about $400 \mathrm{~nm}$ thick.

Small concentration of reactive hydrochloric acid in the electrolyte does not result in fast corrosion of the deposited iron layers. Sodium carbonate solution was added to the cell at the end of the deposition process. Thus, it was possible to remove smooth and shiny layers from the electrolytic cell safely.

When deposition time was too long (deposition time of over $15 \mathrm{~min}$ ) the black coating covered the iron layer, which blocked further iron deposition process. The composition of this coating was examined.

\section{Results of measurements}

\subsection{XPS measurements}

The X-ray photoelectron spectroscopy (XPS) measurements were performed to examine the composition of the black coating. XPS spectra of electrodeposited layers were obtained by a PHI 5700/660 Physical Electronics Photoelectron Spectrometer using a monochromatic $\mathrm{Al} K_{\alpha}$ radiation source $(1486.6 \mathrm{eV})$ operating 
at $15 \mathrm{kV}$. The energy resolution of the instrument was $0.3 \mathrm{eV}$. Just before the measurements a binding energy scale was calibrated within reference to $\mathrm{C} 1 \mathrm{~s}$ $(284.8 \mathrm{eV})$. All the measurements were performed under the UHV conditions. For the measurements of the deeper parts of the sample, the deposited layer was subsequently bombarded by $\mathrm{Ar}^{+}$ion beam with energy $2 \mathrm{keV}$. The results for raw and $\mathrm{Ar}^{+}$sputtered samples are shown in Fig. 1a and Fig. 1b, respectively.

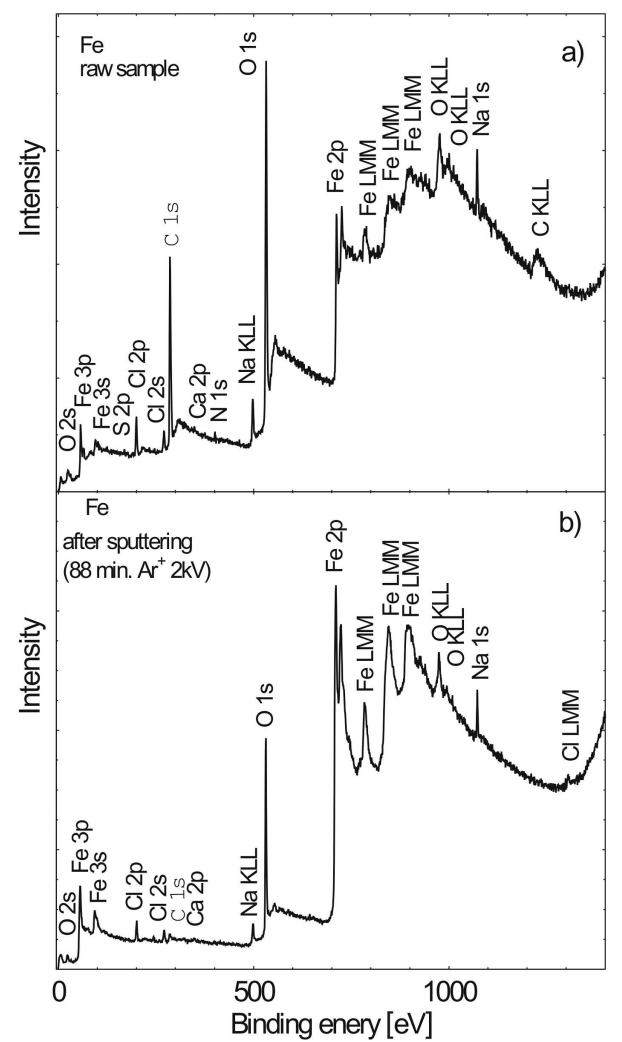

Fig. 1. Photoemission spectra for black coated (a) raw, (b) sputtered for 88 min iron layer surface.

The XPS spectra show that carbon and oxygen dominate on the sample surface. Traces of sodium and chlorine were detected. A decrease in carbon and oxygen peaks' intensities and an increase in photoemission from iron core levels are evident (see Fig. 1) with an increase in the sputtering time. The composition of the layer as a function of sputtering time is presented in Table I.

\subsection{Mössbauer measurements}

The deposited iron layers were examined by conversion electron Mössbauer spectroscopy (CEMS) method. The sample was placed in the detector [10] which allows to carry out measurements with two opposite geometries: the wave vector 
TABLE I

Atomic concentration of $\mathrm{Fe}, \mathrm{C}, \mathrm{O}, \mathrm{Na}$ in the investigated black coated iron layer in a function of sputtering time $t_{\mathrm{sp}}$.

\begin{tabular}{c|c|c|c|c|c|c}
\hline \hline Element & \multicolumn{6}{|c}{ Concentration [\% at.] } \\
\cline { 2 - 7 } & \multicolumn{7}{|c}{$t_{\mathrm{sp}}$} \\
& $0 \mathrm{~min}$ & $10 \mathrm{~min}$ & $20 \mathrm{~min}$ & $50 \mathrm{~min}$ & $70 \mathrm{~min}$ & $88 \mathrm{~min}$ \\
\hline $\mathrm{Fe}$ & 13.1 & 47.2 & 52.1 & 56.8 & 58.4 & 59.3 \\
$\mathrm{C}$ & 43.2 & 5.5 & 2.2 & 0.3 & 0 & 1.2 \\
$\mathrm{O}$ & 41 & 43.0 & 41.8 & 38.7 & 37.2 & 35.2 \\
$\mathrm{Na}$ & 3.2 & 4.3 & 3.9 & 4.3 & 4.4 & 4.3
\end{tabular}

of photon $\boldsymbol{k}$ perpendicular or almost parallel (approximately $2^{\circ}$ ) to the layer plane. All the experiments were performed at room temperature using the spectrometer operating in a constant acceleration mode and a ${ }^{57} \mathrm{Co}$ source in a $\mathrm{Cr}$ matrix. Obtained CEMS spectra for the pure iron layer $\left(t_{\mathrm{D}}=5 \mathrm{~min}\right)$ and the iron layer with black coating $\left(t_{\mathrm{D}}=15 \mathrm{~min}\right)$ are shown in Fig. 2 and Fig. 3, respectively.

The line intensities measured in the Mössbauer experiment for a single nucleus are given by

$$
\begin{aligned}
i_{1}: i_{2}: i_{3}: i_{4}: i_{5}: i_{6} \\
=3\left(1+\cos ^{2} \vartheta\right): 4 \sin ^{2} \vartheta:\left(1+\cos ^{2} \vartheta\right):\left(1+\cos ^{2} \vartheta\right): 4 \sin ^{2} \vartheta \\
\quad: 3\left(1+\cos ^{2} \vartheta\right),
\end{aligned}
$$

where $i_{n}$ refers to the $n$-th line intensity in the sextet and $\vartheta$ is an angle between the directions of the $\boldsymbol{k}$ vector and the hyperfine magnetic field.

As can be seen from (1), in case of magnetic moments arranged in the sample plane, $i_{2} / i_{3}=4$ for the $\boldsymbol{k}$ vector perpendicular to the sample surface, while $i_{2} / i_{3}=4 / 3$ for $\boldsymbol{k}$ parallel to the sample surface. When magnetic moments are arranged perpendicularly to the sample plane: $i_{2} / i_{3}=0$ for $\boldsymbol{k}$ perpendicular and $i_{2} / i_{3}=4$ for $\boldsymbol{k}$ parallel to the sample plane.

The Zeeman sextet (for the iron layer with $t_{\mathrm{D}}=5 \mathrm{~min}$ ) as well as the Zeeman sextet and the doublet (for the iron layer with $t_{\mathrm{D}}=15 \mathrm{~min}$ ) were fitted to the spectra. Hyperfine interaction parameters are presented in Table II. The hyperfine magnetic field $\left(B_{\mathrm{hf}}\right)$ and the isomer shift $(\delta)$, obtained in both cases, are in good agreement with the parameters for $\alpha$-iron $(32.9 \mathrm{~T}$ and $0 \mathrm{~mm} / \mathrm{s}$, respectively). The additional doublet, appearing for the black coated iron layer, is probably due to the iron oxide. The values of the quadrupole splitting $(\Delta)$ and the isomer shift $(\delta)$ are similar to that of wüstite $(\mathrm{FeO})(\Delta=0.98 \mathrm{~mm} / \mathrm{s}$ and $\delta=0.35 \mathrm{~mm} / \mathrm{s}$ [11] $)$ or $\mathrm{Fe}(\mathrm{OH})_{3}(\Delta=0.85 \mathrm{~mm} / \mathrm{s}$ and $\delta=0.36 \mathrm{~mm} / \mathrm{s}[12])$ parameters.

The obtained ratio $i_{2} / i_{3}$ values for measurements with the $\boldsymbol{k}$ vector perpendicular and parallel to the sample surface, for both samples are shown in Table III. 


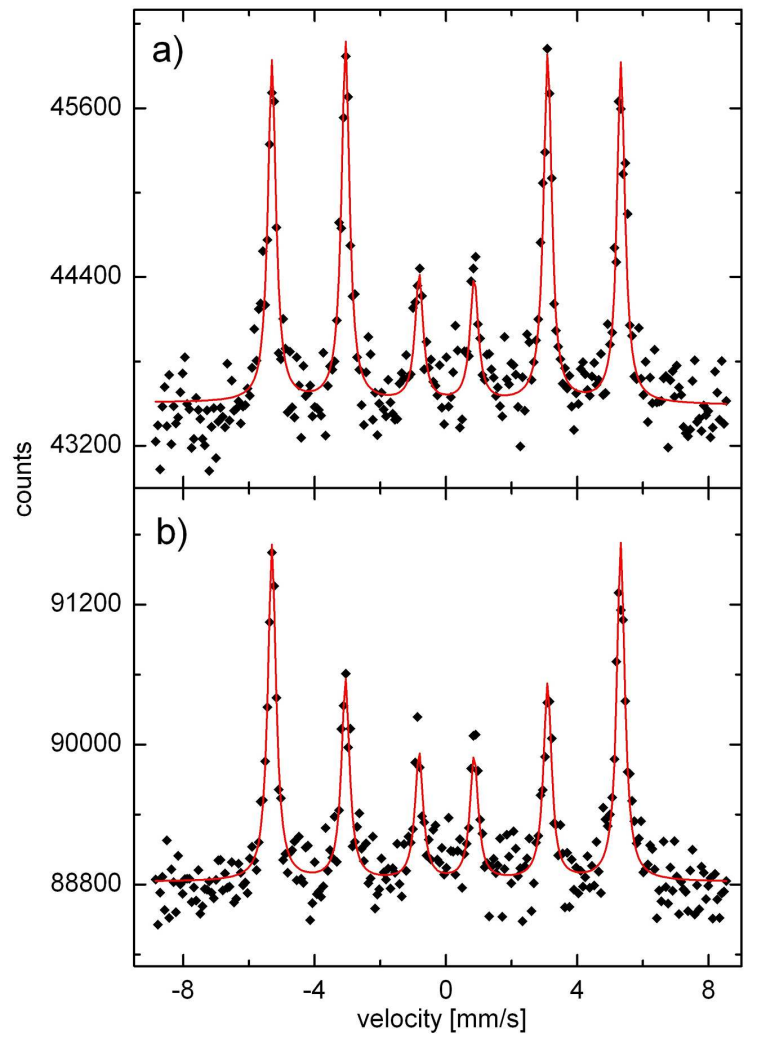

Fig. 2. The CEMS spectrum of a pure iron layer deposited on $\mathrm{Cu}\left(t_{\mathrm{D}}=5 \mathrm{~min}\right)$ and measured with the $\boldsymbol{k}$ vector: (a) perpendicular and (b) almost parallel to the sample surface.

Results obtained for the pure iron layer suggest, on average, in-plane orientation of the magnetic moments, while for the black coated layer indicate that the magnetic moments have small preferential orientation, perpendicular to the sample plane. To highlight changes in magnetic moments arrangement as a function of deposition time, the additional measurements were performed. The results of measurements with the $\boldsymbol{k}$ vector perpendicular to sample surface are shown in Fig. 4.

Apparently, the $i_{2} / i_{3}$ ratio value decreases with increasing deposition time. The value of intensity ratio $(0.98 \pm 0.04)$ obtained for black coated iron layer with the 20 min deposition time (Fig. 4d) strongly deviates from 2 and indicates for perpendicular anisotropy of the black coated iron layer.

\subsection{Magnetization measurements}

The measurements were carried out at room temperature with the use of a vibrating sample magnetometer (VSM) model 7300 Lake Shore in strong (up to $12 \mathrm{kOe}$ ) and weak (up to $50 \mathrm{Oe}$ ) external magnetic field. The obtained magne- 


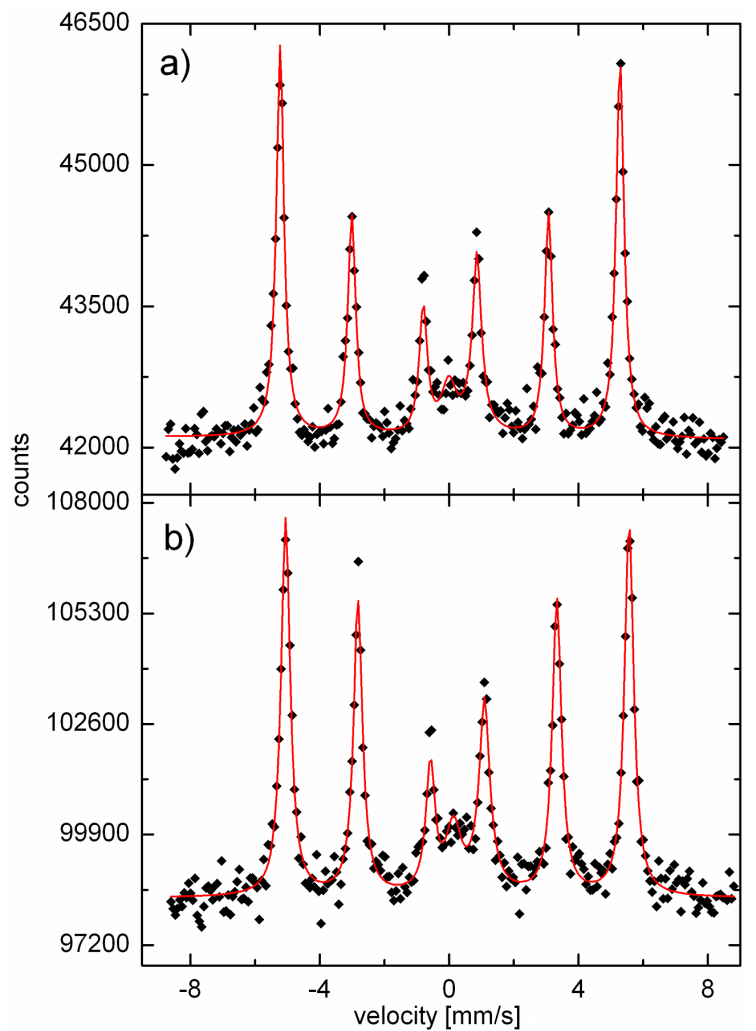

Fig. 3. The CEMS spectrum of a black coated iron layer deposited on $\mathrm{Cu}\left(t_{\mathrm{D}}=15 \mathrm{~min}\right)$ and measured with the $\boldsymbol{k}$ vector: (a) perpendicular and (b) almost parallel to the sample surface.

TABLE II

Hyperfine interaction parameters for electrodeposited iron layers: $B_{\mathrm{hf}}$ - hyperfine field, $\delta$ - isomer shift, $\Delta$ - quadrupole splitting, $k-$ relative contribution of the element.

\begin{tabular}{c|c|c|c|c|c}
\hline \hline$t_{\mathrm{D}}[\mathrm{min}]$ & $B_{\mathrm{hf}}[\mathrm{T}]$ & $\delta[\mathrm{mm} / \mathrm{s}]$ & $\Delta[\mathrm{mm} / \mathrm{s}]$ & $k[\%]$ & Remarks \\
\hline 5 & $32.95 \pm 0.02$ & $0.011 \pm 0.020$ & - & 100 & sextet \\
15 & $32.94 \pm 0.02$ & $0.010 \pm 0.020$ & - & 88 & sextet \\
& - & $0.39 \pm 0.09$ & $0.89 \pm 0.11$ & 12 & doublet
\end{tabular}

tization curves for iron layer $\left(t_{\mathrm{D}}=15 \mathrm{~min}\right)$ are shown in Fig. 5a and Fig. 5b, respectively.

Two additional test samples with a different type of magnetic texture (and the anisotropy) were examined. The first one was a pure $10 \mu \mathrm{m}$ thick iron foil, rolled and annealed $\left(900^{\circ} \mathrm{C}\right.$ for $\left.1 \mathrm{~h}\right)$. The second test sample was a $1 \mathrm{~mm}$ thick 
TABLE III

The ratio of the intensities of the second and the third lines for the iron layers obtained in two geometries of the Mössbauer measurement: the $\boldsymbol{k}$ vector perpendicular and parallel to the sample surface.

\begin{tabular}{c|c|c}
\hline \hline$t_{\mathrm{D}}[\mathrm{min}]$ & $\boldsymbol{k}$ perpendicular to the surface & $\boldsymbol{k}$ parallel to the surface \\
\hline 5 & $3.11 \pm 0.05$ & $1.57 \pm 0.03$ \\
15 & $1.73 \pm 0.04$ & $2.34 \pm 0.06$
\end{tabular}

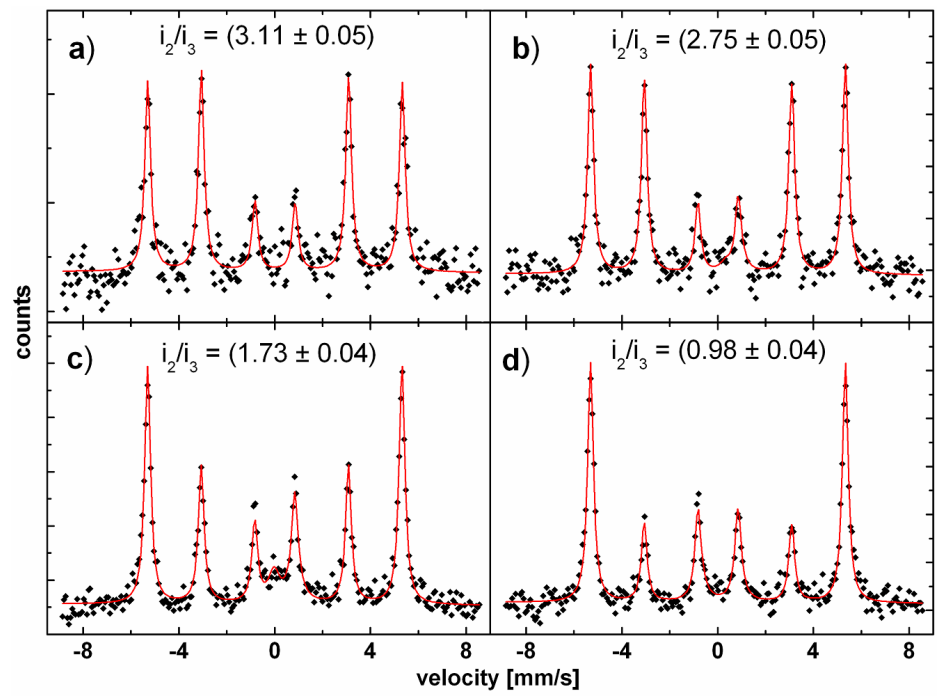

Fig. 4. The Mössbauer spectra of iron layers obtained with different deposition time: (a) $t_{\mathrm{D}}=5 \mathrm{~min},(\mathrm{~b}) t_{\mathrm{D}}=10 \mathrm{~min},(\mathrm{c}) t_{\mathrm{D}}=15 \mathrm{~min}$, (d) $t_{\mathrm{D}}=20 \mathrm{~min}$, measured with the $\boldsymbol{k}$ vector perpendicular to the sample surface.

disk with diameter of $1 \mathrm{~cm}$, containing iron powder with particles' size about few micrometers and nonmagnetic filler $\left(\mathrm{Li}_{2} \mathrm{CO}_{3}\right)$. The iron particles and the filler were mixed with two component liquid resin and exposed to the magnetic field of about $1 \mathrm{~T}$ until solidification of the components. The magnetic field was perpendicular to the disk plane. The procedure results in the creation of the preferred orientation of elongated iron particles. Obtained results are shown in Fig. 6a and Fig. 6b, respectively.

\section{Discussion}

The results of the XPS measurements show that carbon, oxygen and iron are the main components at the top of the layer when electrodeposition time is too long. Traces of sodium and chlorine were also detected, which is expected because sodium carbonate is added to the electrolyte at the end of the process. 

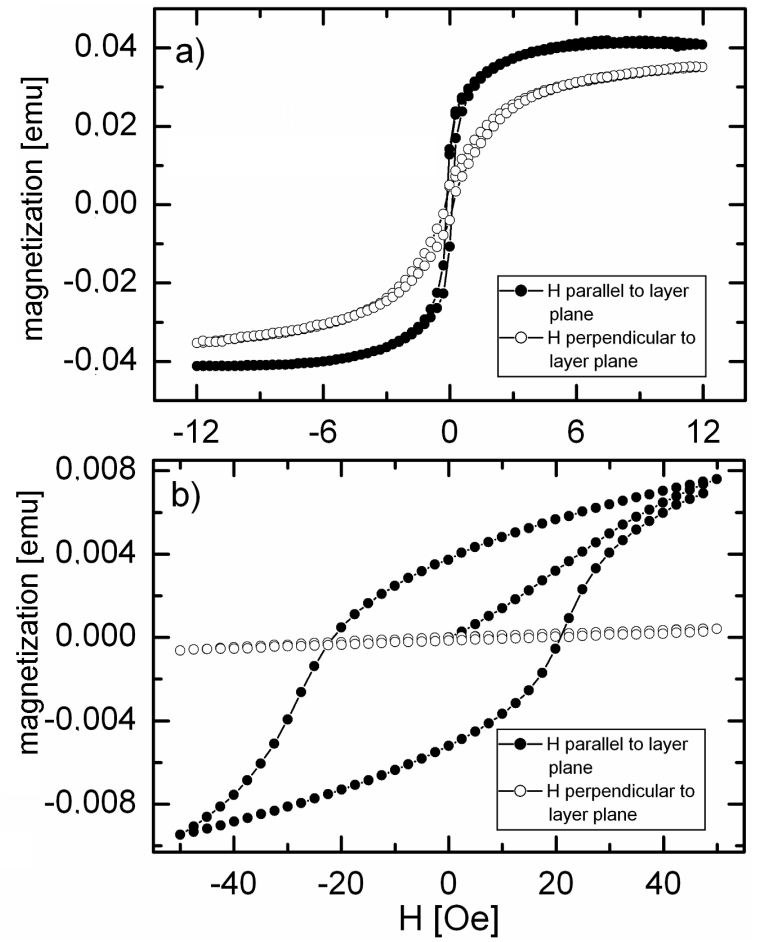

Fig. 5. The magnetization curves for the black coated Fe layer (with $t_{\mathrm{D}}=15 \mathrm{~min}$ ) measured in (a) strong and (b) weak magnetic field.

The CEMS measurements indicate that the magnetic moments' arrangement gradually changes when the deposition time increases and finally the surface of the iron deposit is covered by the black layer. In case of the pure iron layer $\left(t_{\mathrm{D}}=\right.$ 5 min) magnetic moments have, on average, in-plane orientation. In contrast, for the black coated samples $\left(t_{\mathrm{D}}>15 \mathrm{~min}\right)$, magnetic moments' orientation exhibits preferred direction, on average perpendicular to the sample plane. It is clear (Fig. 4) that during the deposition perpendicular anisotropy appears for the black covered samples.

The Mössbauer spectra of the black coated iron layers show small content of the quadrupole doublet. The parameters of the doublet are in good agreement with the parameters of $\mathrm{FeO}$ and/or $\mathrm{Fe}(\mathrm{OH})_{3}$. We cannot exclude that during the electrodeposition process an organic layer (rich in $\mathrm{C}$ and $\mathrm{O}$ - see XPS measurement results in Table I) is forming on the deposited iron and is blocking further iron deposition. This coating contains dispersed particles of wüstite and/or $\mathrm{Fe}(\mathrm{OH})_{3}$ in paramagnetic state.

Results of the magnetization measurements show that the black coated layer $\left(t_{\mathrm{D}}=15 \mathrm{~min}\right)$ has a rather easy-plane type of total anisotropy (see Fig. 5). However, the magnetization curves are considerably different from those measured for 


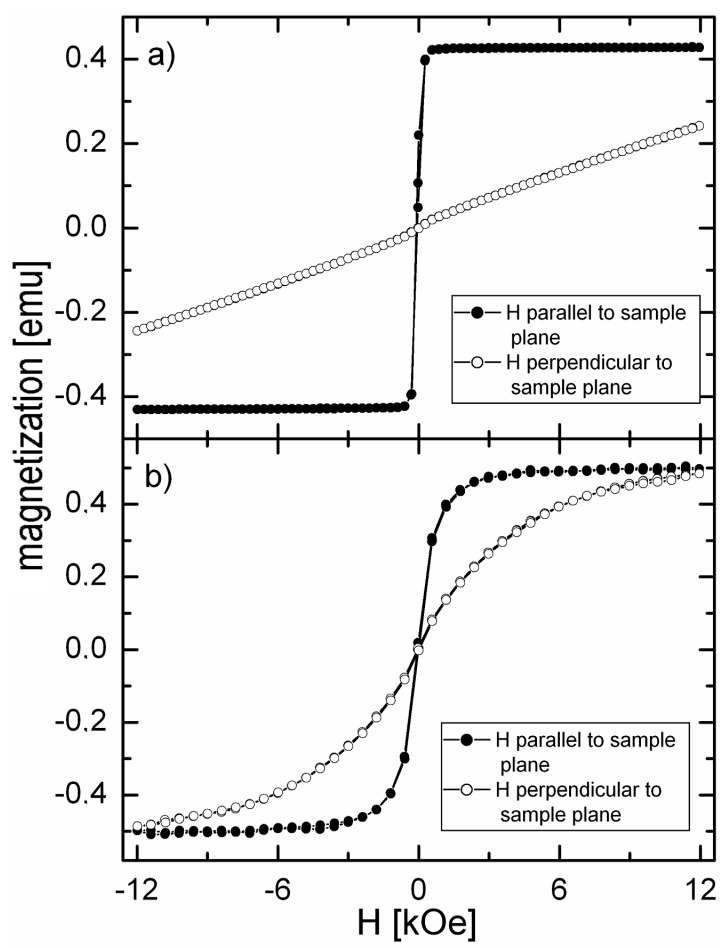

Fig. 6. The magnetization curves for (a) an Fe foil and (b) an iron powder sample oriented in the external magnetic field perpendicular to the sample plane.

the layer of magnetically soft material with clear shape anisotropy. The Fe foil is fully saturated in the magnetic field of only 100 Oe (Fig. 6a), while the deposited layer saturates at field of $6 \mathrm{kOe}$ (Fig. 5a). Thus, the in-plane magnetization processes in the deposited layer are strongly hindered. The possible reason for this behavior is the presence of structural defects formed during the layer growth. The shape of the magnetization curves (Fig. 5a) is similar to the magnetization curves of the sample containing Fe particles oriented in the magnetic field (Fig. 6b). This strongly suggests that in fact there is perpendicular anisotropy in the deposited layer. This conclusion is in full agreement with the results of our CEMS observation.

\section{Summary}

New acetone based electrolyte was used for electroplating of iron layers on copper substrates. The XPS measurements results show high content of carbon in the black coated layer received when the electrodeposition time was too long. The Mössbauer measurements indicate that magnetic moments gradually change their average orientation with deposition time. For short time the moments are oriented in-plane, while for the long deposition time and samples covered by black de- 
posit, magnetic moments show preferential orientation perpendicular to the sample plane. Preferred orientation for long deposition times is evidence of perpendicular anisotropy.

\section{Acknowledgments}

The authors wish to thank Prof. B. Godlewska-Żyłkiewicz from the Institute of Chemistry, University of Białystok, for performing chemical analysis at the initial stage of the project.

\section{References}

[1] L.J. Gao, P. Ma, K.M. Novogradecz, P.M. Norton, J. Appl. Phys. 81, 7595 (1997).

[2] I.W. Wolf, J. Appl. Phys. 33, 1152 (1962).

[3] M. Przybylski, I. Kaufmann, U. Gradmann, Phys. Rev. B 40, 8631 (1989).

[4] Y. Yamada, K. Namiki, Chem. Lett. 36, 294 (2007).

[5] A. Gomes, M.I. da Silva Pereira, M.H. Mendonca, F.M. Costa, Solid State Sci. 4, 1083 (2002).

[6] E. Jartych, D. Chocyk, M. Budzyński, M. Jałochowski, Appl. Surf. Sci. 180, 246 (2001).

[7] Moo Hong Se, Dong Jin Kim, Joung Soo Kim, Thin Solid Films 489, 122 (2005).

[8] E.A. Abd El Meguid, S.S. Abd El Rehim, E.M. Moustafa, Thin Solid Films 443, $53(2003)$

[9] W. Olszewski, K. Szymański, M. Biernacka, R. Sobiecki, Mater. Sci. Poland 26, 743 (2008)

[10] W. Olszewski, K. Szymański, D. Satuła, L. Dobrzyński, L. Bottyán, F. Tanczikó, Nucl. Instrum. Methods Phys. Res. B 266, 3319 (2008).

[11] G.M. da Costa, E. de Grave, P.M.A. de Bakker, R.E. Vanderberghe, J. Solid State Chem. 113, 405 (1994).

[12] S.C.F. Au-Yeung, G. Denes, J.E. Greedan, D.R. Eaton, T. Birchall, Inorg. Chem. 23, 1513 (1984). 